\title{
GWAMAR: Genome-wide assessment of mutations associated with drug resistance in bacteria
}

\author{
Michal Wozniak ${ }^{1 *}$, Jerzy Tiuryn ${ }^{1}$, Limsoon Wong ${ }^{2}$ \\ From The 25th International Conference on Genome Informatics (GIW/ISCB-Asia) \\ Tokyo, Japan. 15-17 December 2014
}

\begin{abstract}
Background: Development of drug resistance in bacteria causes antibiotic therapies to be less effective and more costly. Moreover, our understanding of the process remains incomplete. One promising approach to improve our understanding of how resistance is being acquired is to use whole-genome comparative approaches for detection of drug resistance-associated mutations.

Results: We present GWAMAR, a tool we have developed for detecting of drug resistance-associated mutations in bacteria through comparative analysis of whole-genome sequences. The pipeline of GWAMAR comprises several steps. First, for a set of closely related bacterial genomes, it employs eCAMBer to identify homologous gene families. Second, based on multiple alignments of the gene families, it identifies mutations among the strains of interest. Third, it calculates several statistics to identify which mutations are the most associated with drug resistance.

Conclusions: Based on our analysis of two large datasets retrieved from publicly available data for M. tuberculosis, we identified a set of novel putative drug resistance-associated mutations. As a part of this work, we present also an application of our tool to detect putative compensatory mutations.
\end{abstract}

\section{Background}

The development of drug resistance in bacteria makes antibiotics less effective and increases the costs of therapies. This problem has drawn the attention of major health organizations such as WHO (World Health Organization), ECDC (European Centre for Disease Prevention and Control) and CDC (Centers for Disease Control and Prevention) which monitor and report the epidemics of drug resistance in the world $[1,2]$.

Over a few decades of research on drug resistance, several drug-resistance mechanisms have been discovered such as alteration of a drug's binding site, reduced accumulation of a drug, and specialized enzymes to degrade drug molecules [3]. These mechanisms may be acquired either through chromosomal mutations or horizontal gene transfer.

There are many mutations reported in various studies as associated with drug resistance mechanisms. However,

\footnotetext{
* Correspondence: m.wozniak@mimuw.edu.pl

${ }^{1}$ Faculty of Mathematics, Informatics and Mechanics, University of Warsaw, Warsaw, Poland

Full list of author information is available at the end of the article
}

the information is spread throughout the literature and not easy to access. One attempt to collect the information on genetic features associated with drug resistance into a database is the Antibiotic Drug Resistance Database (ARDB) [4]. However, this database focuses on genes associated with drug resistance rather than particular point mutations within them. Another species-specific database of drug resistance-associated mutations in M. tuberculosis is the Tuberculosis Drug Resistance Mutation Database (TBDReaMDB) [5]. This database provides detailed information on a set of 1230 associations between drugs and point mutations. Furthermore, it distinguishes a subset of high-confidence mutations which were often reported in literature.

The process of acquisition of drug resistance is often associated with some additional cost, called fitness, which reduces the general viability of the bacteria $[6,7]$. However, this effect may be reversed completely or partially by secondary mutations, called compensatory mutations $[7,8]$. Consequently, the evolutionary process of acquiring multiple drug resistance happens in a sequence of steps 
rather than simultaneously [9-11]. Thus, in order to better understand the process, it is important to identify not only the primary mutations responsible directly for drug resistance, but also those secondary mutations allowing for gradual accumulation of drug resistance.

Since the number of bacterial genomes being sequenced and publicly available sequencing data grows rapidly [12], it opens new possibilities for using largescale computational approaches for identifying drug resistance-associated mutations.

In our previous work, we presented an approach to use whole-genome comparative approach for identification of drug resistance-associated mutations [13]. We proposed a new computational scoring scheme, called weighted support, which uses phylogenetic information for identifying the drug resistance-associated mutations. In order to test our approach, we used publicly available sequencing data for 100 strains of Staphylococcus aureus and collected phenotype data from over 70 articles. Our experiment suggested that weighted support outperforms other standard measures such as mutual information and odds ratio.

In the current work, we present GWAMAR, a tool for identifying of drug resistance-associated mutations based on comparative analysis of whole-genome sequences of closely related bacterial strains. This tool is designed as a pipeline. It first employs eCAMBer, our previously published tool [14], to identify point mutations among the set of considered genomes. These mutations constitute the genotype data. Then, GWAMAR tries to find the associations between the input phenotype and genotype data by computing several statistical scores. As a part of this work, we also designed a new statistical score, viz tree-generalized hypergeometric score (TGH).

The rational for incorporating phylogenetic information into TGH and weighted support is based on our observation that subtrees of the phylogenetic tree very often correspond to geographic locations. Since drug resistance mutations are subject to evolutionary pressure caused by the drug treatment they should be independent of geographic location and therefore be more widely distributed over the tree, as opposed to mutations driven by other environmental factors which tend to rather concentrate in small subtrees.

In order to test our approach, we run GWAMAR on two large datasets for $M$. tuberculosis. The first dataset is prepared for the set of 173 strains with genome sequences and annotations publicly available in the PATRIC database [15]. And for this set of strains, we collected drug resistance information from over 20 publications. The genotype and phenotype data for the second dataset comes from the M. tuberculosis Drug Resistance Directed Sequencing Database.

\section{Methods}

We present details of GWAMAR in this section, including the problem setting, the preprocessing of input data and the computation of the association scores between the drug resistance phenotypes and point mutations.

\section{Problem setting}

We consider a set $\mathcal{S}$ of closely related bacterial genomes. Typically, this is a set of strains within the same species of bacteria.

Then, we represent the available drug resistance information as a set of drug-resistance profiles $\mathcal{R}$, where each drug resistance profile $r \in \mathcal{R}$ is represented as a vector:

$$
r: \mathcal{S} \rightarrow\{S, I, R, ?\}
$$

Here, $S, I, R$ denote that a given strain is known to be drug susceptible, intermediate-resistant, or resistant, respectively. Using question mark '?' we indicate that the drug resistance status of a strain is unknown. We call a drug resistance profile complete if it does not contain question marks.

Analogously, we represent the genotype data as a set of mutations $\mathcal{M}$, where each mutation $m \in \mathcal{M}$ is represented as a triple $(g, p, v)$, where $g, p, v$ denote the gene family identifier, the position of the mutation in its corresponding multiple alignment, and the mutation profile, respectively. The mutation profile is represented as a vector:

$$
v: \mathcal{S} \rightarrow \sum \cup\{?\} .
$$

Here $\Sigma$ denotes the set of amino acides (for proteincoding genes) or nucleotides (for promoters and rRNA coding genes). We also assume that $\Sigma$ contains the gap '-' symbol. Using question mark '?' we indicate that the corresponding amino acid or nucleotide is unknown. Analogously, we call a mutation profile complete if it does not contain question marks.

It should be noted that potentially multiple mutations at different positions in the genome may have identical mutation profiles. Moreover, it may happen that multiple mutations may correspond to the same set of mutated strains. In that situation the mutations would essentially carry the same information about the profiles. Thus, we also introduce an auxiliary concept called binary mutation profile. Let $r \in \mathcal{S}$ denote the reference strain and $s \in \mathcal{S}$ denote any strain. Then, for a given mutation profile $v$ its corresponding binary mutation profile is defined as follows:

$$
b(s)=\left\{\begin{array}{l}
? \text { if } \mathrm{v}(\mathrm{s})=? \\
0 \text { if } \mathrm{v}(\mathrm{s})=\mathrm{v}(\mathrm{r}) \\
1 \quad \text { otherwise }
\end{array}\right.
$$


Analogous to mutation profiles, we call a binary mutation profile complete if it does not contain question marks.

Finally, we define the aim of the tool as: To produce an ordered list of associations between the phenotype and genotype data (represented as drug resistance and mutation profiles) such that the top-scored associations are the most likely to be real.

\section{The pipeline of GWAMAR}

Figure 1 illustrates the overall design of the tool. Data preprocessing for GWAMAR consists of several steps which may be performed by our previously developed tool, eCAMBer [14]. These preprocessing steps comprise:

- download genome sequences of multiple bacterial strains,

- unification of gene annotations,

- identification of homologous gene families,

- multiple alignments of the gene families (employing MUSCLE),

- reconstruction of the phylogenetic tree (employing PHYLIP),

- identification of point mutations based on the multiple alignments.

The input drug-resistance profiles, typically, are collected from the articles or databases which provide drug-resistance information for the strains of interest. The set of identified point mutations, the set of drugresistance profiles and and the phylogenetic tree constitute the input for GWAMAR.

In the next step, GWAMAR computes binary mutation profiles for each mutation profile. This step significantly reduces the number of genetic profiles to be scored. Finally, GWAMAR computes several statistical scores to associate drug-resistance profiles to the mutation profiles, including mutual information, odds ratio, hypergeometric test, weighted support (which is our previously published approach [13]), and the tree-generalized hypergeometric score (our new approach here).

\section{Tree-generalized hypergeometric score}

As a part of this work we also introduce a new association score, called tree-generalized hypergeometric score (TGH ). This score is a modification of the CCTSWEEP score introduced in the paper [16]. In this section, we consider a subset of strains $S$ for which a given drugresistance profile $r$ and a binary mutation profile $b$ are complete, i.e. do not contain question marks. Moreover, we assume that $r$ does not contain any intermediateresistant strains. In all our computational experiments we transform the intermediate-resistant strains into resistant strains.
In order to present the formal definition of TGH, we first define an auxiliary concept called coloring. For a given tree $T$, we call a subset $c$ of its nodes a coloring, if it satisfies the following two conditions:

- each path from a leaf to the root contains at most one node from $c$,

- each internal node in $c$ has a sibling node which does not belong to $c$.

Here we also introduce a function $L$ which, for each node $\omega$, returns the set of descendants of the node, including the node itself. We say these nodes are visible from $\omega$. Additionally, the function $L$ applied to a coloring $c$ returns the union of all nodes visible from nodes in $c$.

Let $C_{T}$ denote the set of all colorings of $T$. Then, for each complete drug-resistance profile $r$ there exists exactly one coloring $\hat{c}$ such that the set of leaves visible from $\hat{c}$ equals the set of drug-resistant nodes in $r$. We say this coloring is induced by the drug-resistance profile. Analogously, for each complete binary mutation profile $b$ there is exactly one induced coloring $\bar{c}$.

Intuitively, the coloring induced by a given complete drug-resistance profile will contain the set of nodes in which drug-resistance was acquired (assuming a model in which drug-resistance cannot be reversed). Analogously, the coloring induced by a given binary mutation profile will contain the set of nodes in which the mutation was acquired.

Figure 2 (A) presents an example of colorings induced by a given drug-resistance profile (large red nodes) and a given binary mutation profile (small orange nodes) for a flat tree. In that situation the colorings may be interpreted as independent drawing of balls as in the standard hypergeometric distribution model. Knowing this property of TGH we proposed its name as it generalizes the standard hypergeometric test in the case of a flat tree. Figure 2 (B) presents another example of colorings induced by the same pair of profiles, but for a tree which is not flat. In this model the dependencies between different strains are captured by the topology of the tree.

Let us now assume we want to compute the TGH score for a pair of complete drug-resistance profile $r$ and complete binary mutation profile $b$. Let us additionally assume that the size of coloring $\bar{c}$ induced by $b$ equals $n$. Morover, let the number of nodes in coloring $\bar{c}$ visible from the coloring $\hat{c}$ equals $k$. This value can be interpreted as the number of times the considered mutation was acquired not earlier than the resistance was acquired.

Now, let $V_{T}(n)$ denote the number of colorings of size $n$ :

$$
V_{T}(n)=\#\left\{c \in C_{T}:|c|=n\right\}
$$


The pipeline of GWAMAR

Preprocessing steps done by eCAMBer

(this step may potentially be replaced by other tools)

Download of genome sequences and annotations for a set of bacterial strains

$\checkmark$

Consolidation of genome annotations for multiple bacterial strains and identification of gene families

Multiple alignments of identified gene families computed using MUSCLE

Identification of point mutations
Reconstruction of the phylogenetic tree employing PHYLIP or PhyML

The reference strain

Binarization of mutation

Genotype data (a set of mutations)
Phylogenetic tree for the set of bacterial strains
Phenotype data collected from literature or databases (a set of drug resistance profiles)
Scoring of the mutation Profiles

(multiprocessing)

Scored list of putative associations of drug resistance with mutations

Figure 1 Schema of the GWAMAR pipeline. For a set of considered bacterial strains, the input data for GWAMAR consists of (i) a set of mutations; (ii) a set of drug resistance profiles; and (iii) phylogenetic tree for the set of bacterial strains. Typically the set of mutation profiles is generated using eCAMBer, which is able to download the genome sequences and annotations for the set of bacterial strains, identify point mutations based on multiple alignments, and reconstruct the phylogenetic tree of the considered bacterial strains. The first step of GWAMAR is to compute binary mutation profiles for all point mutations. This step significantly reduces the number of genetic profiles considered. Finally, GWAMAR implements several statistical scores to associate drug resistance profiles with mutation profiles. These include: mutual information, odds ratio, hypergeometric test, weighted support and tree-generalized hypergeometric score (TGH). As a result, we obtain ordered lists of drug resistance associations, where the top scored associations are the most likely to be real. 

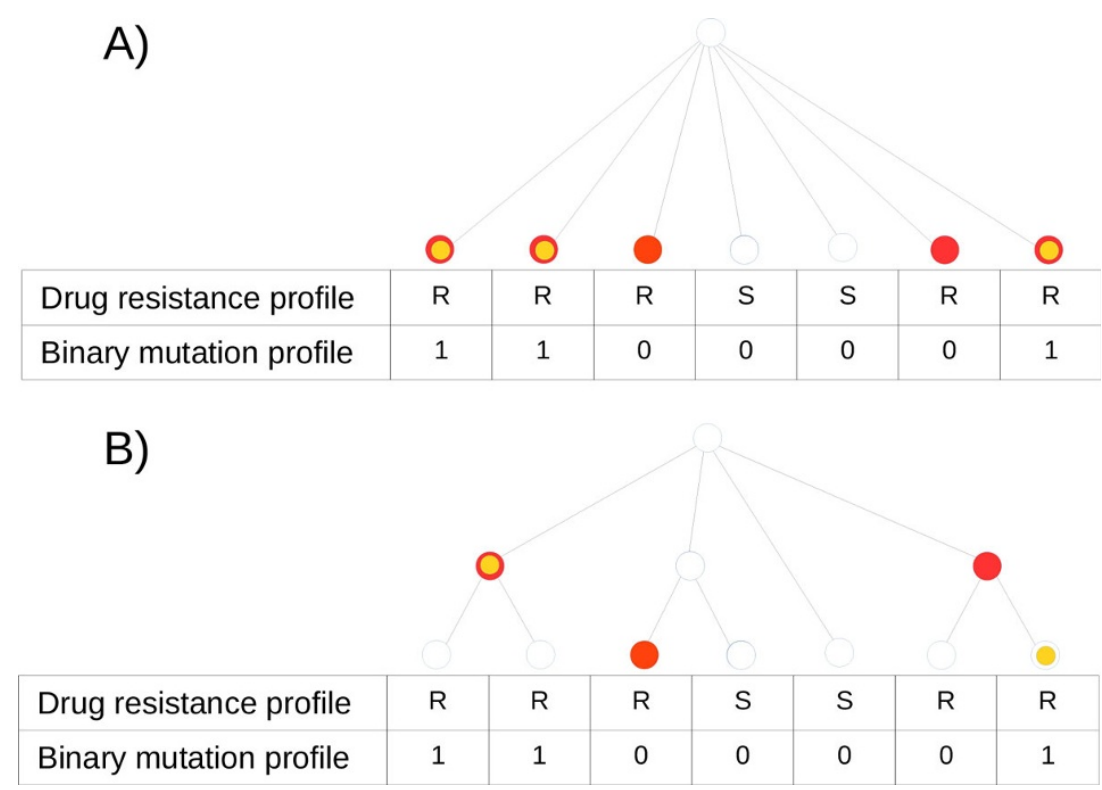

Figure 2 Example colorings for the TGH score. (A) an example of coloring $\hat{c}$ induced by a given drug-resistance profile (large red nodes) and coloring $\bar{c}$ induced by a given binary mutation profile (small orange nodes) for a flat tree. In this example $|\hat{c}|=5,|\bar{c}|=3$ and $|L(\hat{c}) \cap \bar{c}|=3$. (B) another example of colorings $\hat{c}$ and $\bar{c}$ induced by the same pair of profiles but for a different tree. In this example $|\hat{c}|=3,|\bar{c}|=2$ and $|L(\hat{c}) \cap \bar{c}|=2 \mid$

$V_{T}(n)$ may be interpreted as the total number of binary mutation profiles for which the induced coloring is of the same size as for $\bar{c}$.

Then, let $B_{T, \hat{c}}(k, n)$ denote the number of colorings of size $n$, such that exactly $k$ nodes of that coloring are visible from nodes of coloring $\hat{c}$.

$$
B_{T, \hat{c}}(k, n)=\#\left\{c \in C_{T}:|L(\hat{c}) \cap c|=k \text { and }|c|=n\right\}
$$

Here, the value $B_{T, \hat{c}}(k, n)$ may be interpreted as the number of binary mutation profiles such that their induced coloring has $n$ elements, out of which $k$ is visible from the nodes in $\hat{c}$.

Finally, for the complete drug-resistance profile $r$ and complete binary mutation profile $b$, which induce colorings $\hat{c}$ and $\bar{c}$, respectively, we define the TGH score as follows:

$$
H_{T}(r, b)=-\log \left(\frac{\sum_{i=k}^{n} B_{T, \hat{c}}(i, n)}{V_{T}(n)}\right)
$$

Here, we take the negative logarithm to have consistent property for all considered scoring methods, such that the higher the score the more likely drug-resistance profile $r$ is associated with binary mutation profile $b$.

\section{Time complexity}

Let $D$ denote the number of drug-resistance profiles considered. Additionally, let $N$ denote the number of considered strains and $M$ denote the number of binary mutation profiles. Finally, let $K$ denote the maximal number of children of an internal node in the tree. Then, the time complexity of the algorithms we implemented to compute the hypergeometic score, the mutual information, odds ratio, and weighted support is $O(D N M)$. In order to compute $\mathrm{TGH}$, we implement a dynamic programing algorithm which computes the values $B_{\omega, \hat{c}}(k, n)$ for each internal node $\omega, k$ and $n$. This strategy gives an algorithm with complexity $O\left(D \cdot N^{K-1} \cdot N^{2}+D \cdot N \cdot M\right)$. For the brevity of the presentation we skip details of the algorithm.

\section{Results and discussion}

We now present and discuss results we obtained applying GWAMAR on the two datasets we prepared for $M$. tuberculosis.

In all the following analyzes we use the set of mutations classified as high-confidence mutations in Tuberculosis Drug Resistance Mutation Database (TBDReaMDB) as our gold standard [5]. In total the database contains 88 of high-confidence associations spanning 27 genes and six drugs or drug families: Fluoroquinolones, Ethambutol, Isoniazid, Pyrazinamide, Rifampicin and Streptomycin.

\section{Mtu173 case study}

The first case study is based on the set of 173 fully sequenced strains of $M$. tuberculosis with publicly available data.

The preprocessing steps of preparing the genotype data were performed using eCAMBer, our previously 
published tool to support comparative analysis of multiple bacterial strains [13].

In particular, first, we used eCAMBer to download the genome sequences and annotations from the PATRIC database [15]. Next, we applied eCAMBer to unify the genome annotations of protein-coding genes and to identify the clusters of genes with high sequence similarity. Then, for the subset of 4379 such identified gene clusters with genes present in at least $90 \%$ of the strains, we computed multiple alignments using MUSCLE [17]. The multiple alignments were computed for amino-acid sequences of protein-coding genes, as well as nucleotide sequences of their promoter regions, and rRNA genes. Finally, based on the computed multiple alignments, we identified 118913 non-synonymous point mutations. The set of identified point mutations constituted the input genotype data for GWAMAR.

The input phenotype data was collected from over 20 publications issued together with the fully sequenced genomes. Additional file 1 shows the drug-resistance status of the strains retrieved from literature together with references. Based on the drug-resistance information collected for Ciprofloxacin and Ofloxacin, we introduced a new drug-resistance profile for Fluoroquinolones. A strain is categorized as susceptible to Fluoroquinolones if it was susceptible to at least one of the drugs, but not resistant to any of them. Similarly, a strain was categorized as resistant to Fluoroquinolones if it was resistant to at least one of the drugs, but not susceptible to any of them. If none of the cases was satisfied for a strain, then the drug-resistance status of the strain was categorized as unknown. We restrict further analysis to the set of six drugs or drug families: Fluoroquinolones, Ethambutol, Isoniazid, Pyrazinamide, Rifampicin and Streptomycin.

The input phylogenetic tree was reconstructed using the maximum-likelihood approach implemented in the PHYLIP package. As an input for the tool we used the set of all the identified mutations concatenated into one multiple alignment file. Additional file 2 presents the reconstructed phylogenetic tree.

Table 1 presents the list of top 20 associations ordered according to TGH score. In the set of 20 top-scoring associations, 15 are present in the TBDReaMDB database and 13 of them are categorized as high-confidence mutations. A closer look at the mutations which are not present in TBDReaMDB revealed that some of them can be supported by literature. In particular, mutation E504G/D in embB has recently been reported as associated with resistance to Ethambutol [18]. Similarly, the mutation T539I has already been associated with resistance to Fluoroquinolones [19].

Literature search did not provide us any additional support for the remaining three mutations (A505T in

Table 120 top-scoring associations between drug-resistance profiles and point mutations in the case study on 173 fully sequenced M.tuberculosis strains.

\begin{tabular}{|c|c|c|c|c|c|c|}
\hline drug name & gene id & gene name & mutation & all & h.c. & TGH \\
\hline Fluoroquinolones & Rv0006 & gyrA & D94G/A/H/N/Y & Y & Y & 14.1843430424 \\
\hline Isoniazid & Rv1908c & katG & S315T/G/N & Y & Y & 9.04507605888 \\
\hline Rifampicin & Rv0667 & rpoB & S450L & Y & Y & 8.60191917013 \\
\hline Streptomycin & Rv0682 & rpsL & K43R & Y & Y & 8.32303955124 \\
\hline Ethambutol & Rv3795 & embB & M306I/N/L & Y & Y & 8.24966301883 \\
\hline Isoniazid & Rv1483 & fabG1 & $\mathrm{C}-15 \mathrm{~T}$ & Y & Y & 5.8445976648 \\
\hline Rifampicin & Rv0667 & rpoB & $\mathrm{D} 435 \mathrm{~F} / \mathrm{Y} / \mathrm{G} / \mathrm{A}$ & Y & Y & 5.0402225732 \\
\hline Streptomycin & Rv0682 & rpsL & K88R/M & Y & Y & 4.16354931535 \\
\hline Ethambutol & Rv3795 & embB & E504G/D & $N$ & $N$ & 3.33103155053 \\
\hline Pyrazinamide & Rv2043c & pncA & W68L & Y & Y & 2.7080502011 \\
\hline Pyrazinamide & Rv2043c & pncA & H51P & Y & Y & 2.7080502011 \\
\hline Rifampicin & Rv0667 & rpoB & $\mathrm{H} 445 \mathrm{D} / \mathrm{Y} / \mathrm{R}$ & Y & Y & 2.52993515037 \\
\hline Streptomycin & Rvnr01 & rrs & G1108C & $N$ & $N$ & 1.71691080314 \\
\hline Ethambutol & Rv3795 & embB & D1024N & Y & $\mathrm{N}$ & 1.68763546921 \\
\hline Ethambutol & Rv3795 & embB & D869G & $N$ & $N$ & 1.68763546921 \\
\hline Ethambutol & Rv3795 & embB & A505T & $N$ & $N$ & 1.68763546921 \\
\hline Fluoroquinolones & Rv0005 & gyrB & N538T & Y & Y & 1.68478734968 \\
\hline Fluoroquinolones & Rv0006 & gyrA & S91P & Y & Y & 1.68478734968 \\
\hline Fluoroquinolones & Rv0005 & gyrB & T539| & $N$ & $N$ & 1.68478734968 \\
\hline Streptomycin & Rvnr01 & rrs & A1401G & Y & $N$ & 1.28846347057 \\
\hline
\end{tabular}

Each row corresponds to one association, whereas the consecutive columns describe: drug name, gene identifier, gene name, mutation, association presence in the TBDReaMDB database, status indicating if the association is categorized as high confidence in TBDReaMDB, TGH score. 
embB, D869G in embB and G1108C in rrs), which haven't been reported in TBDReaMDB. These mutations may potentially be false positives or real drug-resistanceassociated mutations.

\section{Mtu_broad case study}

The second case study, mtu_broad, is based on the data available in the Broad Institute database http://www. broadinstitute.org/annotation/genome/mtb_drug_resistance.1/. This database contains provides sequencing data and drug-resistance information for 1398 strains of M. tuberculosis. However, it should be noted that only genes of interest were sequenced. Table 2 presents the list of 28 sequenced genes for each strain. Additionally 12 promoter sequences were sequenced. In total, this database contains 1069 mutations (non-synonymous aminoacid changes or nucleotide changes in promoters).

Similar to the previous case study, based on the drugresistance information available in the database for Ciprofloxacin, Ofloxacin, Levofloxacin and Moxifloxacin, we introduced a new drug-resistance profile for Fluoroquinolones. A strain was categorized as susceptible to Fluoroquinolones if it was susceptible to at least one of the drugs, but not resistant to any of them. Similarly, a strain was categorized as resistant to Fluoroquinolones if it was resistant to at least one of the drugs, but not susceptible to any of them. If none of the cases was satisfied for a strain, then the drug-resistance status of the strain was categorized as unknown. We restrict further analysis to the set of six drugs or drug families: Fluoroquinolones, Ethambutol, Isoniazid, Pyrazinamide, Rifampicin and Streptomycin.

Similarly, as in the previous case study, the phylogenetic tree was reconstructed using the maximum-likelihood approach implemented in the PHYLIP package. As an input for the tool we used the set of all available mutations concatenated into one multiple alignment file.

Table 3 presents the list of the top 20 associations ordered according to TGH score. In the set of 20 topscoring associations, 19 are present in TBDReaMDB and 15 of them are categorized as high-confidence mutations.

A closer look at the mutation D89G/N, which is not present in TBDReaMDB, reveals that the mutation has recently been associated with resistance to Fluoroquinolones [20].

The set of associations provides some additional support for the four mutations which were present in TBDReaMDB, but not categorized as high-confidence.

Table $\mathbf{2}$ List of sequenced genes and promoters available in the $\mathbf{m t u}$ broad dataset.

\begin{tabular}{|c|c|c|c|}
\hline gene id & gene name & description & promoter sequenced? \\
\hline Rv0005 & gyrB & DNA gyrase subunit $B$ & yes \\
\hline Rv0006 & gyrA & DNA gyrase subunit $A$ & yes \\
\hline Rv0341 & iniB & isoniazid inductible gene protein & yes \\
\hline Rv0342 & iniA & isoniazid inductible gene protein & yes \\
\hline Rv0343 & inic & isoniazid inductible gene protein & yes \\
\hline Rv0667 & rров & DNA-directed RNA polymerase beta chain & yes \\
\hline Rv0682 & rpsL & $30 S$ ribosomal protein $\mathrm{S} 12$ & yes \\
\hline Rv1483 & fabG1 & 3-oxoacyl-[acyl-carrier protein] reductase & yes \\
\hline Rv1484 & inhA & NADH-dependent enoyl-[acyl-carrier-protein] reductase & yes \\
\hline Rv1694 & tlyA & cytotoxin-haemolysin & no \\
\hline Rv1854C & ndh & NADH dehydrogenase & yes \\
\hline Rv1908c & katG & catalase-peroxidase-peroxynitritase $T$ & no \\
\hline Rv2043c & pncA & pyrazinamidase/nicotinamidas & yes \\
\hline Rv2245 & kasA & 3-oxoacyl-[acyl-carrier protein] synthase 1 & no \\
\hline Rv2427Ac & oxyR' & hypothetical protein & no \\
\hline Rv2428 & ahpC & alkyl hydroperoxide reductase $C$ protein & yes \\
\hline Rv2764c & thyA & thymidylate synthase & yes \\
\hline Rv2764c & ddl & D-alanine-D-alanine ligase ddlA & no \\
\hline Rv3423c & alr & alanine racemase & no \\
\hline Rv3793 & $\mathrm{embC}$ & membrane indolylacetylinositol arabinosyltransferase & yes \\
\hline Rv3794 & embA & membrane indolylacetylinositol arabinosyltransferase & yes \\
\hline Rv3795 & embB & membrane indolylacetylinositol arabinosyltransferase & yes \\
\hline Rv3854c & ethA & monooxygenase & yes \\
\hline Rv3919c & gid & glucose-inhibited division protein B & yes \\
\hline Rvnr01 & rrs & ribosomal RNA $16 \mathrm{~S}$ & no \\
\hline Rvnr02 & $\mathrm{rrl}$ & ribosomal RNA 235 & no \\
\hline
\end{tabular}


Table 320 top-scoring associations between drug-resistance profiles and point mutations in the case study for 1398 partially sequenced M.tuberculosis strains.

\begin{tabular}{|c|c|c|c|c|c|c|}
\hline drug name & gene id & gene name & mutation & all & h.c. & TGH \\
\hline Fluoroquinolones & Rv0006 & gyrA & D94A/G/N/Y/H & Y & $Y$ & 129.754964792 \\
\hline Fluoroquinolones & Rv0006 & gyrA & A90G $N$ & Y & Y & 41.8967753922 \\
\hline Streptomycin & Rv0682 & rpsL & K43R & Y & Y & 31.005838239 \\
\hline Isoniazid & Rv1908c & katG & $\mathrm{S} 315 \mathrm{~T} / \mathrm{S} / \mathrm{G} / \mathrm{N} / \mathrm{l} / \mathrm{R}$ & Y & Y & 27.1918713598 \\
\hline Ethambutol & Rv3795 & embB & Q497P/R/K/H & Y & Y & 17.1681425414 \\
\hline Streptomycin & Rv0682 & rpsL & $\mathrm{K} 88 \mathrm{~T} / \mathrm{R} / \mathrm{Q} / \mathrm{M}$ & Y & Y & 16.2806822989 \\
\hline Fluoroquinolones & Rv0005 & gyrB & N538K $/$ /D/S & Y & Y & 12.6368065275 \\
\hline Rifampicin & Rv0667 & rpoB & $\mathrm{H} 445 \mathrm{P} / \mathrm{D} / \mathrm{R} / \mathrm{Y} / \mathrm{L} / \mathrm{N} / \mathrm{Q}$ & Y & Y & 12.627849397 \\
\hline Streptomycin & Rvnr01 & rrs & A1401G & Y & N & 9.60726487825 \\
\hline Pyrazinamide & Rv2043c & pncA & T135P/A & Y & $\mathrm{N}$ & 9.35766011848 \\
\hline Streptomycin & Rvnr01 & rrs & A514C & Y & Y & 8.96892262877 \\
\hline Rifampicin & Rv0667 & rpoB & $\mathrm{D} 435 \mathrm{Y} / \mathrm{N} / \mathrm{H} / \mathrm{G} / \mathrm{A} / \mathrm{N}$ & Y & Y & 7.63431166207 \\
\hline Fluoroquinolones & Rv0006 & gyrA & S91P & Y & Y & 7.57935978224 \\
\hline Pyrazinamide & Rv2043c & pncA & $\mathrm{T}-11 \mathrm{C} / \mathrm{G}$ & Y & Y & 6.76727069266 \\
\hline Ethambutol & Rv3795 & embB & G406S/D/A/C & Y & Y & 6.32500852932 \\
\hline Fluoroquinolones & Rv0006 & gyrA & D89G/N & $\mathrm{N}$ & $\mathrm{N}$ & 6.26814578901 \\
\hline Pyrazinamide & Rv2043c & pncA & L120P/R & Y & $N$ & 6.11085770664 \\
\hline Streptomycin & Rvnr01 & rrs & C517T & Y & Y & 5.16411345885 \\
\hline Ethambutol & Rv3795 & embB & $\mathrm{D} 328 \mathrm{Y} / \mathrm{G} / \mathrm{H}$ & Y & N & 5.07901609928 \\
\hline Pyrazinamide & Rv2043c & pncA & V139G/A/M/L & Y & Y & 5.05727324518 \\
\hline
\end{tabular}

This dataset is provided by The Broad Institute. Each row corresponds to one association, whereas the consecutive columns describe: drug name, gene identifier, gene name, mutation, association presence in the TBDReaMDB database, status indicating if the association is categorized as high confidence in TBDReaMDB, TGH score.

\section{Assessment of accuracy}

Here we use the two datasets described above to assess the accuracy of the various association scores, viz: mutual information, odds ratio, hypergeometric score, weighted support and TGH. The CCTSWEEP software might contain some bugs and did not produce correct output. Its authors had not responded to our queries in time. So we omitted it from our experiments.

As our gold standard we use the set of 88 drug-resistance associations classified as high-confidence in the Tuberculosis Drug Resistance Mutation Database (TBDReaMDB) [5].

In both case studies, as the set of positives, we assume the subset of high-confidence mutations present in TBDReaMDB, which are also present in the genotype data. This is the set of mutations which may be potentially detected using the available datasets. There are 39 and 75 of such associations for the mtu173 and mtu_broad datasets, respectively. The set of negatives is constructed by random sampling from the whole set of identified putative associations except for the associations which are classified as positives. The number of sampled negatives equals the total number of mutations present in genes which has at least one high-confidence mutation. We use this approach in order to significantly reduce the probability of classifying as negatives associations which are real but not present in the database.
Figure 3 presents precision and recall curves for different association scores. Consistently, results on both datasets suggest that tree-aware scores outperform treeignorant scores.

\section{Rifampicin resistance and compensatory mutations}

It is commonly known that Rifampicin resistance in $M$. tuberculosis gets acquired by point mutations within the RRDR region which corresponds to the Rifampicin binding spot [21]. However, these mutations are often associated with deleterious effect on bacteria fitness [22]. This effect may be potentially reversed by compensatory mutations. Recently, three new papers have been released, focusing on identifying putative compensatory mutations within rpoA, rpoB and rpoC genes [23-25].

Figure 4 presents the distribution of the putative compensatory mutations identified in these recent studies, put together with mutations identified based on our two case studies.

Interestingly, several mutations identified by our approach have also been reported in at least one of the papers [23-25].

- rpoA: G31S/A

- rpoB: P45S/L, L731P, E761D, R827C, H835P/R

- rpoC: G332R/S, V431M, G433C/S, V483G/A, W484G, I491T/V, L527V, N698K, A734V 


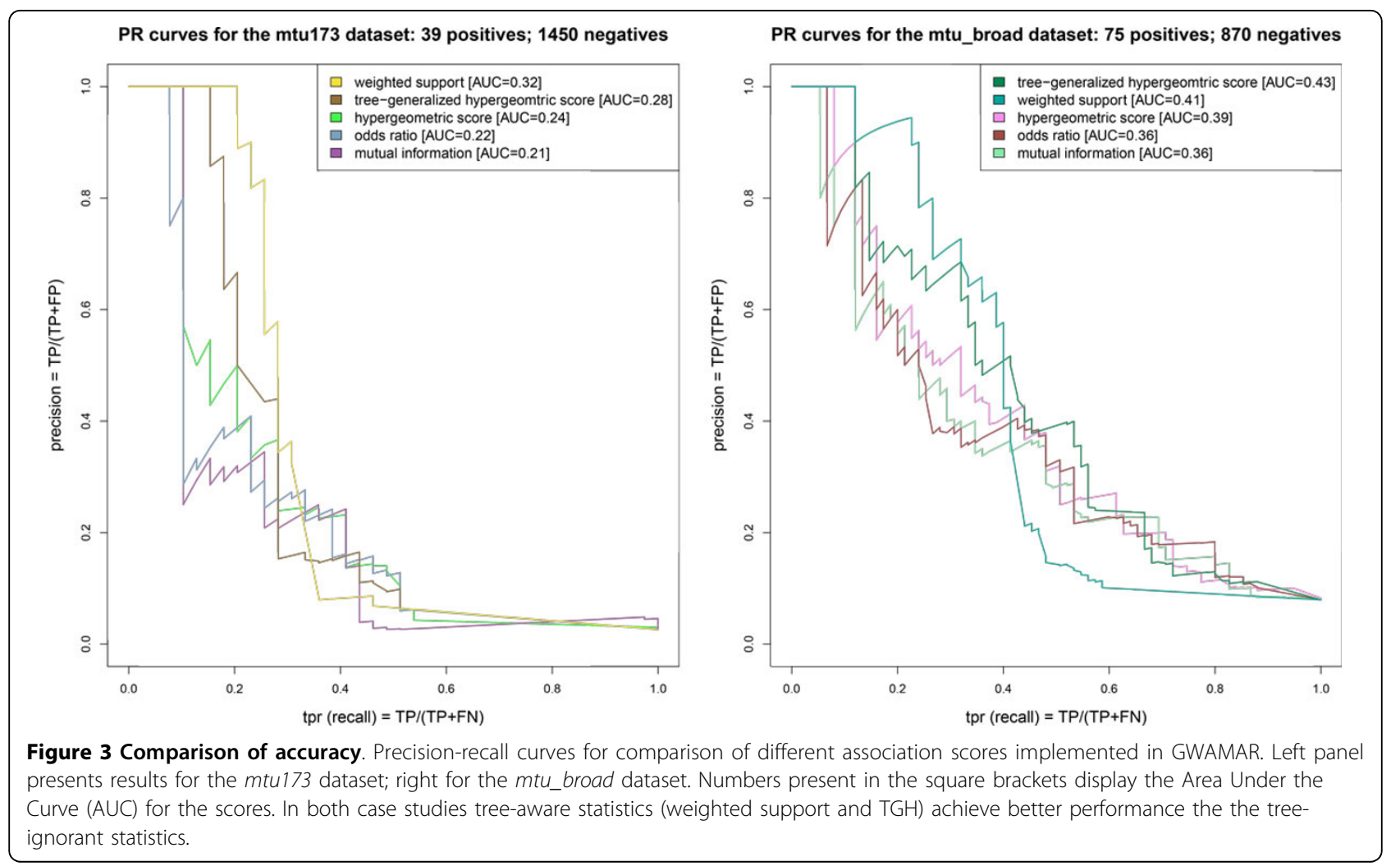

Additional file 3 contains the detailed table with the complete list of putative compensatory mutations.

\section{Conclusion}

In this work we presented GWAMAR, a tool we have developed for identifying of drug-resistance associated mutations based on comparative analysis of whole-genome sequences in bacterial strains.

The tool is designed as an automatic pipeline which employs eCAMBer, our previously published tool [14], for preprocessing of data. This preprocessing includes: (i) download of genome sequences and gene annotations,

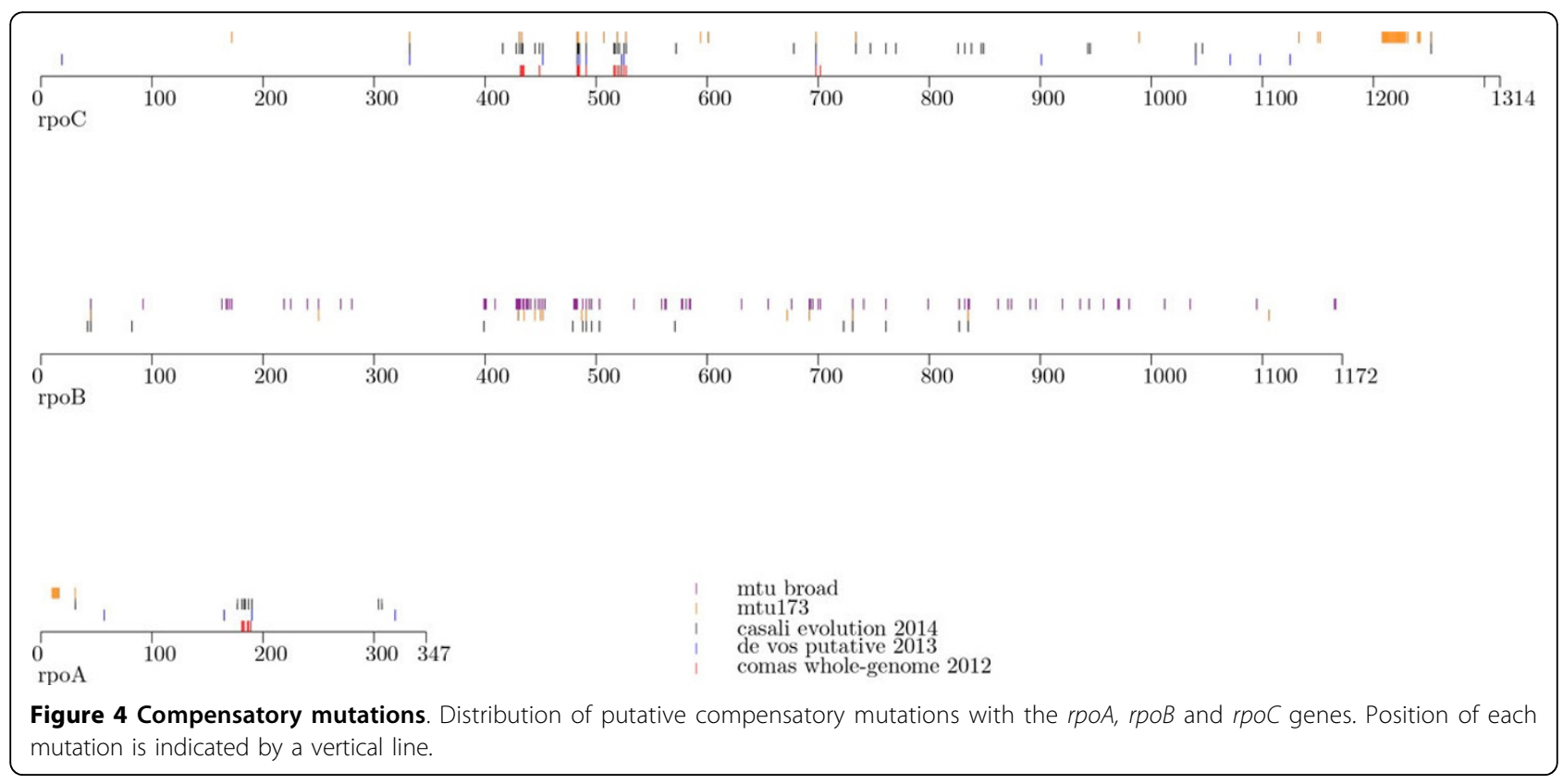


(ii) unification of gene annotations among the set of considered strains, (iii) identification of gene families, (iv) computation of multiple alignments and identification of point mutations which constitute the input genotype data.

GWAMAR implements various statistical methodssuch as mutual information, odds ratio, hypergeometric test and weighted support [13]- to associate the drugresistance phenotypes with point mutations. As a part of this work, we also present tree-generalized hypergeometric score (TGH) - a new statistical score.

In order to test our approach, we prepared two datasets for M. tuberuclosis. Results of both case studies suggest that tree-aware methods (weighted support and TGH) perform better than methods which do not incorporate phylogenetic information. This result supports also our corollary from our previous paper about weighted support [13]. Employing GWAMAR on the two datasets, we identified novel mutations putatively associated with drug-resistance.

Finally, despite some promising results, the presented tool has some limitations. First, it does not take into account or predict epistatic interactions between mutations. Second, it only takes into account genomic changes ignoring levels of gene expression. Thirdly, it provides putative in-silico associations which should be subjected to further investigation in wet lab experiments.

\section{Availability}

The GWAMAR software, input data and results of the case study experiments are available at the website: http://bioputer.mimuw.edu.pl/gwamar.

\section{Additional material}

Additional file 1: Drug-resistance information for 173 strains of $M$. tuberculosis. Drug-resistance information collected from literature for 173 strains of $M$. tuberculosis.

Additional file 2: Phylogenetic tree for 173 strains of $M$. tuberculosis. Phylogenetic tree reconstructed using the maximum-likelihood method implemented in the PHYLIP for 173 strains of M. tuberculosis.

Additional file 3: Putative compensatory mutations. Complete list of putative compensatory mutations identified in three different studies and our two datasets.

\section{Competing interests}

The authors declare that they have no competing interests.

\section{Authors' contributions}

All authors contributed to design of the method, analysis of results and writing of the manuscript. MW wrote software and performed experiments. All authors read and approved the final manuscript.

\section{Acknowledgements}

This work is supported in part by Polish Ministry of Science and Higher Education grant no. 2012/05/ST6/03247 and Singapore Ministry of Education Tier-2 grant no. MOE2009-T2-2-004.

\section{Declarations}

Funding for the publication of this paper was provided by Wong's KITHCT chair professorship.

This article has been published as part of BMC Genomics Volume 15 Supplement 10, 2014: Proceedings of the 25th International Conference on Genome Informatics (GIW/ISCB-Asia): Genomics. The full contents of the supplement are available online at http://www.biomedcentral.com/ bmcgenomics/supplements/15/S10.

\section{Authors' details}

${ }^{1}$ Faculty of Mathematics, Informatics and Mechanics, University of Warsaw, Warsaw, Poland. ${ }^{2}$ School of Computing, National University of Singapore, Singapore.

Published: 12 December 2014

\section{References}

1. WHO | Antimicrobial resistance: global report on surveillance 2014. 2014 [http://www.who.int/drugresistance/documents/surveillancereport/en/], Accessed 2014-07-12.

2. Threat Report 2013 | Antimicrobial Resistance | CDC (2013). [http://www. cdc.gov/drugresistance/threat-report-2013/], Accessed 2014-07-12.

3. Wright GD: Molecular mechanisms of antibiotic resistance. Chemical Communications (Cambridge, England) 2011, 47(14):4055-4061.

4. Liu B, Pop M: ARDB-antibiotic resistance genes database. Nucleic Acids Research 2009, 37(Database):443-447.

5. Sandgren A, Strong M, Muthukrishnan P, Weiner BK, Church GM, Murray MB: Tuberculosis drug resistance mutation database. PLOS Med 2009, 6(2):1000002, Accessed 2014-07-12.

6. Andersson Dl, Hughes D: Antibiotic resistance and its cost: is it possible to reverse resistance? Nature Reviews Microbiology 2010, 8(4):260-271.

7. Koch A, Mizrahi $V$, Warner DF: The impact of drug resistance on mycobacterium tuberculosis physiology: what can we learn from rifampicin? Emerging Microbes \& Infections 2014, 3(3):17, Accessed 2014-07-14.

8. Wiesch PSz, Engelstädter J, Bonhoeffer S: Compensation of fitness costs and reversibility of antibiotic resistance mutations. Antimicrobial Agents and Chemotherapy 2010, 54(5):2085-2095, Accessed 2014-07-14.

9. Palmer $A C$, Kishony R: Understanding, predicting and manipulating the genotypic evolution of antibiotic resistance. Nature Reviews Genetics 2013, 14(4):243-248, Accessed 2014-07-14.

10. Toprak E, Veres A, Michel JB, Chait R, Hartl DL, Kishony R: Evolutionary paths to antibiotic resistance under dynamically sustained drug selection. Nature Genetics 2012, 44(1):101-105, Accessed 2014-07-14

11. Angst DC, Hall AR: The cost of antibiotic resistance depends on evolutionary history in escherichia coli. BMC Evolutionary Biology 2013, 13(1):163, Accessed 2014-07-14.

12. Loman NJ, Constantinidou C, Chan JZM, Halachev M, Sergeant M, Penn CW, Robinson ER, Pallen MJ: High-throughput bacterial genome sequencing: an embarrassment of choice, a world of opportunity. Nature Reviews Microbiology 2012, 10(9):599-606.

13. Wozniak M, Tiuryn J, Wong L: An approach to identifying drug resistance associated mutations in bacterial strains. BMC Genomics 2012, 13(Suppl 7):23, Accessed 2014-06-26.

14. Wozniak M, Wong L, Tiuryn J: eCAMBer: efficient support for large-scale comparative analysis of multiple bacterial strains. BMC Bioinformatics 2014, 15(1):65, Accessed 2014-07-14.

15. Wattam AR, Abraham D, Dalay O, Disz TL, Driscoll T, Gabbard JL, Gillespie JJ, Gough R, Hix D, Kenyon R, Machi D, Mao C, Nordberg EK, Olson R, Overbeek R, Pusch GD, Shukla M, Schulman J, Stevens RL, Sullivan DE, Vonstein V, Warren A, Will R, Wilson MJC, Yoo HS, Zhang C, Zhang Y, Sobral BW: PATRIC, the bacterial bioinformatics database and analysis resource. Nucleic Acids Research 2014, 42(D1):581-591, Accessed 2014-07-12.

16. Habib F, Johnson AD, Bundschuh R, Janies D: Large scale genotypephenotype correlation analysis based on phylogenetic trees. Bioinformatics (Oxford, England) 2007, 23(7):785-788.

17. Edgar RC: MUSCLE: multiple sequence alignment with high accuracy and high throughput. Nucleic Acids Research 2004, 32(5):1792-1797, Accessed 2014-07-14.

18. Bakuł AZ, Napió , Rkowska A, Bielecki J, Augustynowicz-Kopeć Ewa, Zwolska Z, Jagielski T: Mutations in the embB gene and their association 
with ethambutol resistance in multidrug-resistant Mycobacterium tuberculosis clinical isolates from poland. BioMed Research International 2013, 2013:167954, Accessed 2014-07-14.

19. Malik S, Willby M, Sikes D, Tsodikov OV, Posey JE: New insights into fluoroquinolone resistance in mycobacterium tuberculosis: Functional genetic analysis of gyrA and gyrB mutations. PLOS ONE 2012, 7(6):39754, Accessed 2014-07-14.

20. Li J, Gao X, Luo T, Wu J, Sun G, Liu Q, Jiang Y, Zhang Y, Mei J, Gao Q: Association of gyrA/b mutations and resistance levels to fluoroquinolones in clinical isolates of mycobacterium tuberculosis. Emerging Microbes \& Infections 2014, 3(3):19, Accessed 2014-07-14.

21. Patra SK, Jain A, Sherwal BL, Khanna A: Rapid detection of mutation in RRDR of rpo $b$ gene for rifampicin resistance in MDR-pulmonary tuberculosis by DNA sequencing. Indian journal of clinical biochemistry: IJCB 2010, 25(3):315-318.

22. Brandis G, Hughes D: Genetic characterization of compensatory evolution in strains carrying rpoB ser531leu, the rifampicin resistance mutation most frequently found in clinical isolates. Journal of Antimicrobial Chemotherapy 2013, 68(11):2493-2497, Accessed 2014-07-15.

23. Comas I, Borrell S, Roetzer A, Rose G, Malla B, Kato-Maeda M, Galagan J, Niemann S, Gagneux S: Whole-genome sequencing of rifampicin-resistant mycobacterium tuberculosis strains identifies compensatory mutations in RNA polymerase genes. Nature Genetics 2012, 44(1):106-110, Accessed 2014-07-14.

24. de Vos M, Muller B, Borrell S, Black PA, van Helden PD, Warren RM, Gagneux S, Victor TC: Putative compensatory mutations in the rpoC gene of rifampin-resistant mycobacterium tuberculosis are associated with ongoing transmission. Antimicrobial Agents and Chemotherapy 2013, 57(2):827-832, Accessed 2014-07-14.

25. Casali N, Nikolayevskyy V, Balabanova Y, Harris SR, Ignatyeva O, Kontsevaya I, Corander J, Bryant J, Parkhill J, Nejentsev S, Horstmann RD, Brown T, Drobniewski F: Evolution and transmission of drug-resistant tuberculosis in a russian population. Nature Genetics 2014, 46(3):279-286, Accessed 2014-07-14.

doi:10.1186/1471-2164-15-S10-S10

Cite this article as: Wozniak et al:: GWAMAR: Genome-wide assessment of mutations associated with drug resistance in bacteria. BMC Genomics 2014 15(Suppl 10):S10.

\section{Submit your next manuscript to BioMed Central and take full advantage of:}

- Convenient online submission

- Thorough peer review

- No space constraints or color figure charges

- Immediate publication on acceptance

- Inclusion in PubMed, CAS, Scopus and Google Scholar

- Research which is freely available for redistribution

Submit your manuscript at www.biomedcentral.com/submit
Biomed Central 